\title{
La urgencia psiquiátrica en un hospital general. La patología de la agresividad principal motivo de consulta.
}

\author{
Psychiatric emergencies in a general hospital. \\ Agressiveness as a main consultation motive.
}

\begin{abstract}
RESUMEN: Análisis descriptivo de la urgencia psiquiátrica en un hospital general durante 6 años. Se mantienen como grupo principal los pacientes psicóticos, se detecta un aumento de pacientes con trastornos de personalidad. El principal motivo de consulta es la agresividad. Predominan mujeres con conductas autolesivas y varones con conductas violentas.

PALABRAS CLAVE: urgencia psiquiátrica, agresividad, autolesiones, hospital general.
\end{abstract}

\begin{abstract}
Retrospective study of the cases attended by the psychiatric emergency service in a general hospital during 6 years. Psychotic patients represent the main group, and there is an increase in patients with personality disorders. The main consultation topics were self/ hetero agressiveness, specially autolesive women and males showing agressive behaviour.

KEY WORDS: psychiatric emergency, aggressiveness, autolesive, general hospital.
\end{abstract}

\section{Introducción.}

En los últimos años hemos asistido a un importante desarrollo de los servicios de Salud Mental. Entre ellos se encuentran los dispositivos de atención de urgencias psiquiátricas integrados en el servicio de urgencias general, como respuesta a un incremento de las demandas por una mayor sensibilidad sanitaria y social a los problemas de Salud Mental. Es importante contar con datos que dimensionen cuantitativa y cualitativamente las características de esta demanda para el diseño de los servicios de urgencias y la asignación de recursos humanos. En el presente trabajo ofrecemos un análisis descriptivo de la demanda urgente atendida por el equipo de Psiquiatría de urgencias en el Hospital Virgen del Rocío de Sevilla (HVR), con una población asignada como hospital de referencia de 875.331 habitantes según datos de la memoria año 2003.

Los Hospitales Universitarios "Virgen del Rocío" son un complejo hospitalario formado por 4 hospitales en un mismo recinto: Hospital Infantil, Hospital de la Mujer, Hospital de Rehabilitación y Traumatología, y Hospital General (HG), con un total de 1571 camas instaladas. En el HG se encuentra ubicada la Unidad de Hospitalización Psiquiátrica, con una capacidad de 28 camas, y en el servicio de urgencias general se atienden las urgencias psiquiátricas en una consulta específica de Psiquiatría. La media de urgencias/día en HG es de 322 en el año 2003, las urgencias psiquiátricas son atendidas durante $24 \mathrm{~h} /$ día por un médico especialista en Psiquiatría de los servicios de Salud Mental del área hospitalaria, 
acompañado por un médico residente de Psiquiatría o en su defecto de Medicina Familiar y Comunitaria en su periodo de rotación por Salud Mental. Las urgencias psiquiátricas provienen de: pacientes externos que acuden motu propio o derivados por otras instancias sanitarias o sociales al servicio de urgencias; tras valoración de triage se asigna al psiquiatra directamente o son derivados tras valoración clínica por los médicos del servicio de urgencia HG y de los servicios de urgencias de los otros 3 hospitales. Tan bien provienen de interconsultas urgentes de pacientes ingresados en todo el HVR; y por último de pacientes ingresados en la unidad de hospitalización psiquiátrica.

Entre los psiquiatras y médicos residentes de Psiquiatría, que cubrían la urgencia, existía la opinión de que se estaba produciendo un aumento de la cantidad de demandas y un cambio cualitativo con un menor número de pacientes psicóticos y un mayor número de pacientes con trastorno de personalidad, problemas de drogas, conductas violentas. El trabajo pretende contrastar la veracidad de esa opinión.

En la revisión bibliográfica hemos encontramos numerosos estudios dedicados a aspectos concretos de las urgencias, estudian determinados grupos de casos según patología, destacando los dedicados al suicidio. Nuestro estudio tiene el interés de registrar la totalidad de la demanda recibida y de poder complementar esas informaciones parciales.

Son dos los objetivos principales, conocer las características de los pacientes atendidos y en segundo lugar investigar su evolución y sus tendencias en estos 6 años. Estos objetivos salieron de la opinión extendida entre los psiquiatras de guardia acerca de que se estaba produciendo un incremento cuantitativo de las urgencias psiquiátricas y se estaba produciendo una diferencia cualitativa, con un aumento de pacientes con trastornos de la personalidad y una disminución de paciente psicóticos.

\section{Metodología}

La muestra la componen pacientes atendidos por el equipo de urgencias psiquiátricas de HVR entre 1999 y 2003. El número de intervenciones psiquiátricas de urgencias en un año se mantiene cercano a las 5000 intervenciones. Los datos de 1999 a 2002 fueron extraídos del "libro de guardia", documento de registro clínico en donde se anotan diariamente todos los casos atendidos por el equipo de urgencias psiquiátricas. La definición de la muestra se realizo escogiendo aleatoriamente de forma proporcional guardias de 24 horas de 8 a.m. a 8 a.m. del día siguiente. Se distribuyeron proporcionalmente meses, semanas y días de 
ORIGINALES Y REVISIONES

la semana, de los años 1998, 1999, 2000, 2001, 2002. Los datos del año 2003 se obtienen sobre la asistencia directa rellenando una hoja de registro de los casos atendidos durante los meses de marzo a junio.

Se diseñó específicamente una hoja de registros de datos para el presente estudio. Se estudian las variables: sexo, hora de asistencia, día de la semana, origen de la demanda, motivo de consulta, diagnóstico sindrómico, intervención realizada y destino del paciente Los registros fueron realizados por psiquiatras y residentes de Psiquiatría, que conocían los criterios de recogida de datos y los consensos del grupo de investigación. El tamaño de la muestra permite un nivel de confianza del 95,5. Para el análisis de los datos se ha usado el programa estadístico SPSS versión 12.

\section{Resultados.}

3.1 Frecuencia de asistencia. (tabla1)

Respecto a la frecuencia casos/día por años no se observa una distribución regular de incremento. La media de 7.25 es la menor frecuencia en el año 2000, y 11.19 la mayor en el año 1998. La media del total asistencias/día es de 9.1. Valoramos que un porcentaje pequeño ha podido quedar sin registrar en el libro, ya que este es independiente de las historias clínicas y tramites administrativos, siendo la media de 9,1 y el máximo de asistencias registrados en un día de 26 y el de menor frecuencia de 3.

3.2 Frecuencia por día de la semana. (Tabla 2)

No se observa una diferencia estadísticamente significativa en la distribución de la asistencia, siendo el día con más asistencia el sábado, con una media de 9.81, y el de menor frecuencia el viernes, con 8.18 de media.

3.3 Horario de asistencia (tabla 3)

No encontramos significatividad estadística en su distribución a lo largo de los años, se mantiene estable su distribución. El periodo de mayor asistencia es el horario de tarde (entre las 15,00 horas y las $22,00 \mathrm{~h}$ ), con un $42 \%$ de la asistencia total. En el turno de mañana se produce un $26.8 \%$ de la asistencia, similar al periodo de noche, con un $24.8 \%$. El periodo con una menor frecuencia de asistencias es la madrugada, con un $5.5 \%$ de las intervenciones.

3.4 Distribución por sexo. (tabla 4)

En su distribución se mantiene estable a lo largo de los años estudiados, sin significación estadística. Es mayor el porcentaje de varones (55.7\%) respecto al de las mujeres (44.3\%). Esta relación está invertida en los estudios de consultas generales ambulatorias de salud mental, con mayor porcentaje de mujeres en 
torno al $60 \%$.

3.5 Origen de la demanda. (tabla 5)

La distribución también se mantiene estable a lo largo de los años estudiados, siendo el paciente que proviene del servicio de urgencias general el origen de la mayoría de las intervenciones, un $78 \%$ de los casos atendidos. La unidad de hospitalización psiquiátrica es el segundo proveedor de, a gran distancia, con un $8.9 \%$. El tercero, las interconsultas urgentes de pacientes ingresados en otros servicios del HVR, el 6.6\% de las asistencias. Por último, la asistencia a pacientes hospitalizados en el área de urgencias (observación y estancias cortas) supone el $5.2 \%$ de las asistencias.

\subsection{Motivo de consulta. (tabla 6)}

En la hoja de registro se permitía indicar en un paciente más de un motivo de consulta, por lo que analizamos los resultados considerando tanto el $\%$ de respuestas como el \% de los casos. En una cuarta parte de los casos no se pudo determinar con claridad el motivo de consulta, según el registro clínico del libro de guardia.

Encontramos que el motivo de consulta más frecuente es el relacionado con intentos o gestos suicidas, presente en un $18.3 \%$ de los casos y que supone el $16.7 \%$ de las respuestas. El segundo motivo más frecuente lo constituyen los trastornos de conducta, que incluyen agresividad y riesgo de violencia, presentes en el $11.4 \%$ de los casos y que suponen el $10.4 \%$ de las respuestas. El tercer motivo, a muy poca distancia del anterior, son los estados de ansiedad y crisis de angustia con un $11.3 \%$ de los casos y un $10.3 \%$ de las respuestas. El cuarto lugar lo ocupa los estados delirantes y/o alucinatorios con un $9.4 \%$ de los casos y un $8.6 \%$ de las respuestas.

3.7 Diagnóstico sindrómico. (tabla 7)

Se asignó cada paciente a uno de los cuadros sindrómicos más frecuentes en la urgencia psiquiátrica, y se registró en cada asistencia a un único grupo, a pesar de que en casos de patología dual cumple los criterios para ambos, o en casos de patología afectiva y psicótica. En estos casos el investigador optó por decidir cual era el diagnóstico psiquiátrico principal independiente del motivo de consulta. En un $22 \%$ de los casos no se asignó a ningún grupo diagnóstico al entender que no había datos registrados suficientes para asignarlo, aunque el libro de guardia incluye el diagnóstico de los casos atendidos.

El grupo diagnóstico mas frecuente lo han constituido los pacientes con síndrome psicótico, con un $20.7 \%$ de los casos. A continuación, la mayor frecuencia se da en pacientes calificados con síndromes neuróticos en que predominan los problemas de ansiedad, que supone el $14.7 \%$ de los casos, seguidos muy de cerca por los pacientes con un síndrome caracterial perteneciente a los trastornos de la personalidad, que suponen el 14\%. Los pacientes cuyo principal problema era la 
ORIGINALES Y REVISIONES

dependencia a tóxicos suponen el cuarto grupo más numeroso, con un $8.9 \%$ de frecuencia.

3.8 Destino del paciente. (tabla 8)

Se mantiene estable a lo largo del tiempo la estructura de derivación del paciente, no hay significación estadística en su evolución. La derivación a un servicio de Salud Mental ambulatorio es la resolución mas frecuente en nuestras urgencias psiquiátricas, con un $37 \%$ de los casos. La hospitalización psiquiátrica con un $19 \%$ de los casos, supone la segunda medida más frecuente, siendo fundamentalmente voluntario su carácter, excepto en torno al 5\%, que presenta el carácter de involuntario (datos de 2003). El 14\% de los casos atendidos continúan ingresados tras la intervención, representan el trabajo de interconsulta, en el que esta incluida la actuación en la sala de psiquiatría. En torno al 5\% se presentan varios destinos, fugas principalmente del área de urgencias, derivación del paciente a atención primaria sin necesidad de tratamiento especializado, traslado a otro hospital para ingreso psiquiátrico debido a la existencia de otra unidad de hospitalización en el área y ser el HVR hospital de referencia en la zona. El traslado a otra especialidad, presente en un 5.2\% de los casos, es debido a las derivaciones para valoración y tratamiento de patología orgánica.

\section{Discusión.}

Después de la revisión bibliográfica pensamos que el interés de nuestro trabajo principalmente reside en presentar una muestra completa, lo que nos ofrece una visión general de las urgencias psiquiátricas. La muestra caracteriza las urgencias psiquiátricas que acuden a un hospital general, dentro de un sistema sanitario que integra la salud mental junto al resto de especialidades médicas. El mayor número de artículos encontrados abordan aspectos parciales de las urgencias. Los pacientes con conductas autolesivas son el grupo más estudiado, también la patología dual, los psicóticos, pacientes sin hogar, trastornos de personalidad.

La respuesta que hemos obtenido a las dos preguntas que planteamos inicialmente: 1 . no observamos en nuestro estudio un incremento de las urgencias que se han mantenido más o menos estables a lo largo de estos años, y 2. las características clínicas de las urgencias presentan ligeras modificaciones en los seis años estudiados, se mantienen estables sus características generales de motivos de consulta y tipo de pacientes. Un mayor número de pacientes diagnosticados de trastorno de personalidad y un mayor número de urgencias relacionadas con drogas serían las dos tendencias que marcan la diferencia. 
La distribución de sexo en la muestra presenta similitud con las registradas en otros estudios (1) aproximándose a nuestros porcentajes coincidiendo en un ligero predominio de varones, resultados coincidentes con los obtenidos en estudios (2) (3) sobre urgencias en un Centro de Salud Mental Comunitario de nuestra misma área de asistencia. Otros estudios encuentran porcentajes en torno al $50 \%$. Como un aspecto colateral varios trabajos se ocupan de valorar la incidencia de la luna llena en la frecuencia o característica de las urgencias psiquiatritas, coincidiendo en no encontrar influencia. (4).

4.1 ¿Por qué consultan?

Si sumamos los dos grupos más frecuentes, autolisis $(18.3 \%)$ y conductas agresivas (11.4\%), suponen el 29,7\% convirtiéndose en el principal motivo con diferencia respecto al siguiente, los estados de ansiedad que suponen el $11 \%$. Es decir los motivos de consulta que presentan en primer plano las conducta agresivas o violentas, ya sea contra uno mismo o contra otros, suponen la principal causa por lo que es consultado el psiquiatra en urgencias. El cuarto motivo son crisis psicóticas que suponen el 10\%. Así, la agresividad supone el principal síntoma de alarma para la consulta urgente psiquiátrica.

Las conductas autolíticas han sido frecuentemente estudiadas (5), presentan una frecuencia similar a nuestro trabajo, en torno al $15 \%$ en estudios en España y Argentina (6) (7). La actuación recomendada en todos los trabajos es la valoración psiquiátrica tras la atención médica general, lo que se lleva a cabo de forma protocolizada en nuestro hospital.

La patología de la agresividad se convierte así en el principal motivo de consulta psiquiátrica de urgencias. En un estudio realizado en Argentina (8) que aborda específicamente este aspecto de la urgencia psiquiátrica se encuentra las conductas violentas en un 38\% de los casos. La asociación de carencia de hogar, abuso de drogas y violencia es estudiada como casos con hiperfrecuentación y que exigen una respuesta de diferentes servicios asistenciales coordinados (9).

El tercer motivo en frecuencia ha sido los estados de ansiedad, ya sea crisis o síndrome ansioso. En otros estudios presentan un mayor porcentaje de esta patología, probablemente debido a que en nuestro caso los médicos de urgencias generales atienden la patología ansiosa y solo derivan al psiquiatra los casos relevantes. En cambio, la práctica totalidad de la patología psicótica o afectiva que se detecta en el área de urgencias es derivada para valoración por el psiquiatra, y los pacientes con riesgo de autolisis.

Si analizamos la variable sexo en los casos que consultan por trastornos de conducta-agresividad, autolisis y abuso de drogas, su distribución por sexo presenta diferencias claramente significativas, las mujeres suponen el doble que los varones en los intentos de autolisis y los varones consultan el doble que las mujeres por problemas de droga y conductas agresivas (Tabla 9). 
ORIGINALES Y REVISIONES

En un estudio reciente en España se mantiene esta distribución por sexos significativa (6) $35,9 \%$ varones y $64,1 \%$ mujeres con parasuicidio, también ratificado en un estudio belga (10). Un estudio canadiense (11) estudia las actitudes violentas en la consulta encontrando una distribución similar en hombres y mujeres, aunque lo que varía es la forma de expresión de la violencia, verbal en las mujeres y física en los hombres, otros estudios no encuentran diferencias significativas entre sexos en las conductas violentas (12).

\section{2 ¿Quienes consultan?}

Una de las preguntas que nos hicimos era si se estaba produciendo un cambio en la patología de los consultantes de urgencias. La consulta de los pacientes psicóticos se mantiene estable a lo largo de los años (en torno al 20\%) y se ha producido, sobre todo en los dos últimos años estudiados, unos incrementos de pacientes que presentan un síndrome caracterial, que llega a igualarse prácticamente porcentualmente al número de pacientes psicóticos que acuden. Si los pacientes psicóticos suponen un $20.9 \%$ de los casos, en cambio el motivo de consulta relacionado directamente con un cuadro psicótico agudo es solo de un $9.4 \%$ de los casos. Es decir, que hay otros motivos de consulta en los pacientes psicóticos además de las crisis psicóticas. Está descrito el uso de urgencias como servicio de Salud Mental ambulatorio para pacientes deteriorados o carentes de recursos sociales.

Los pacientes diagnosticados de síndrome caracterial-trastorno de personalidad, sufren un incremento en los dos últimos años revisados y se igualan a los pacientes psicóticos en número. Coincide este aumento con menos porcentaje de pacientes no diagnosticados, por lo que podría ser efecto de una valoración diagnóstica mas apurada sobre los casos de estos años.

Nuestras conclusiones coinciden en líneas generales con otros autores como Teijeiro (13) que encuentra un aumento de pacientes diagnosticados como "patología dual" y trastornos de personalidad, y no se presentan variaciones significativas respecto a otros diagnósticos psiquiátricos. Este interés por lo pacientes diagnosticados de trastorno de la personalidad va en aumento en los últimos años, un ejemplo lo tenemos en un reciente estudio realizado en Jaén que se ocupa monográficamente de los casos de esta patología que acuden a urgencia (14). Los pacientes psicóticos se mantienen estables en su frecuentación y suponen aproximadamente un tercio en el estudio mencionado (13), nuestro resultado es similar si sumamos las psicosis afectivas y los cuadros delirantes-alucinatorios con el resultado de un $31,9 \%$.

4.3 Destino del paciente

La derivación a un servicio de Salud Mental ambulatorio es la resolución mas frecuente de la urgencia psiquiátrica, en un $37 \%$ de los casos. Hemos de tener en cuenta que los casos no psiquiátricos son derivados previamente por 
triaje al médico internista. El ingreso psiquiátrico, con un 19\% de los casos, supone la segunda medida, y coincide con los resultados de un hospital similar de Barcelona (15) con un 20\% de ingresos. El 14\% de los casos atendidos continúan ingresados, por lo que representan la medida exacta del trabajo de interconsulta, en el que incluimos la actuación en la sala de psiquiatría. En torno al 5\% presentan otros destinos, fugas mientras esperan la valoración del psiquiatra, derivación del paciente a atención primaria sin necesidad de tratamiento especializado, y el traslado a otro hospital para ingreso psiquiátrico debido a la existencia de otra unidad de hospitalización en el área. Traslado a otra especialidad significa que se deriva fundamentalmente a Medicina Interna para descartar patología orgánica o porque el psiquiatra presupone una patología orgánica en juego.

Nos llama la atención que el servicio está orientado hacia la respuesta clínica tanto por el filtro previo hasta llegar al psiquiatra (16) como por la derivación a recursos sanitarios especializados en la gran mayoría de los casos. Para problemas de urgencias ligadas a crisis psicosociales no hay respuesta organizada, solo están cubiertas las respuestas clínicas.

\section{Conclusiones.}

Como resultados destacables encontramos:

Ligero predominio de varones sobre mujeres (60/40)

Las conductas manifiestas de auto o heteroagresividad suponen el principal motivo de consulta urgente psiquiátrica $(29,7 \%)$. La distribución por sexo de estas conductas presenta diferencias significativas: Predominio de varones con trastornos de conducta (heteroagresividad) y de mujeres con conductas autolíticas.

La mayor frecuencia de asistencia en horario de tarde, y el sábado el día de mayor asistencia.

El 75\% de los casos son pacientes externos que acuden al servicio de Urgencias. El 25\% son pacientes ingresados en otros servicios clínicos del hospital.

La mayoría se derivan para seguimiento en Centro de Salud Mental (37\%), y un $19 \%$ ingresan en la unidad de hospitalización psiquiátrica.

Se mantiene estable, en los 6 años estudiados, el porcentaje de psicóticos que consultan $(20,9 \%)$, apreciando un aumento en los trastornos de personalidad y los problemas de abuso de drogas.

El perfil del paciente que acude a una urgencia psiquiátrica es principalmente un varón que consulta por conducta de agresividad, o una mujer por conductas autolesivas. 


\section{ORIGINALES Y REVISIONES}

Tabla 1

Media casos día/año

\begin{tabular}{llllllll}
\hline Año & 1998 & 1999 & 2000 & 2001 & 2002 & 2003 & Total \\
\hline Casos registrados & 403 & 383 & 399 & 360 & 351 & 1021 & 2917 \\
\hline Días & 36 & 46 & 55 & 47 & 42 & 98 & 324 \\
\hline $\begin{array}{l}\text { Media } \\
\text { pacientes/día }\end{array}$ & 11,19 & 8,32 & 7,25 & 7,65 & 8,35 & 10,42 & 9,1 \\
\hline
\end{tabular}

Tabla 2

Número de casos/ días de la semana

\begin{tabular}{llllllll}
\hline Lunes & Martes & Miércoles & Jueves & Viernes & Sábado & Domingo & Media \\
\hline 13,60 & 12,20 & 9,00 & 9,80 & 11,00 & 11,17 & 11,60 & 11,19 \\
\hline 10,00 & 9,50 & 7,38 & 6,43 & 9,60 & 9,17 & 7,38 & 8,32 \\
\hline 5,10 & 7,13 & 10,33 & 5,38 & 5,11 & 10,71 & 9,29 & 7,25 \\
\hline 8,17 & 8,50 & 7,75 & 7,83 & 6,38 & 9,00 & 6,57 & 7,74 \\
\hline 8,60 & 11,40 & 8,83 & 7,86 & 6,75 & 7,33 & 9 & 8,53 \\
\hline 9,21 & 8,00 & 11,87 & 11,29 & 10,33 & 10,38 & 10,75 & 10,40 \\
\hline 8,7 & 9,45 & 9,56 & 8,62 & 8,18 & 9,81 & 9,13 & 9 \\
\hline
\end{tabular}

Tabla 3

Horario de asistencia/año

\begin{tabular}{|c|c|c|c|c|c|c|c|c|}
\hline & & \multicolumn{6}{|l|}{ Año } & \multirow[t]{2}{*}{ Total } \\
\hline & & 1998 & 1999 & 2000 & 2001 & 2002 & 2003 & \\
\hline Mañana & Recuento & 118 & 110 & 106 & 91 & 94 & 224 & 743 \\
\hline $8-15$ & $\%$ de año & $29,3 \%$ & $28,7 \%$ & $26,6 \%$ & $25,3 \%$ & $26,8 \%$ & $25,7 \%$ & $26,8 \%$ \\
\hline Tarde & Recuento & 155 & 172 & 152 & 141 & 144 & 415 & 1179 \\
\hline $15-22$ & $\%$ de año & $38,5 \%$ & $44,9 \%$ & $38,1 \%$ & $39,2 \%$ & $41,0 \%$ & $47,5 \%$ & $42,6 \%$ \\
\hline Noche & Recuento & 113 & 84 & 119 & 97 & 88 & 187 & 688 \\
\hline $22-04$ & $\%$ de año & $28,0 \%$ & $21,9 \%$ & $29,8 \%$ & $26,9 \%$ & $25,1 \%$ & $21,4 \%$ & $24,8 \%$ \\
\hline Madrugada & Recuento & 17 & 17 & 22 & 28 & 25 & 43 & 152 \\
\hline 04-08 & $\%$ de año & $4,2 \%$ & $4,4 \%$ & $5,5 \%$ & $7,8 \%$ & $7,1 \%$ & $4,9 \%$ & $5,5 \%$ \\
\hline Total & Recuento & 403 & 383 & 399 & 360 & 351 & 873 & 2769 \\
\hline
\end{tabular}


Tabla 4

Sexo/año

\begin{tabular}{|c|c|c|c|c|c|c|c|c|c|}
\hline & & & \multicolumn{6}{|l|}{ Año } & \multirow[t]{2}{*}{ Total } \\
\hline & & & 1998 & 1999 & 2000 & 2001 & 2002 & 2003 & \\
\hline \multirow[t]{4}{*}{ Sexo } & Mujer & Recuento & 186 & 184 & 183 & 141 & 153 & 368 & 1215 \\
\hline & & $\%$ de año & $46,5 \%$ & $49,1 \%$ & $46,0 \%$ & $40,6 \%$ & $43,8 \%$ & $42,3 \%$ & $44,3 \%$ \\
\hline & Varón & Recuento & 214 & 191 & 215 & 206 & 196 & 503 & 1525 \\
\hline & & $\%$ de año & $53,5 \%$ & $50,9 \%$ & $54,0 \%$ & $59,4 \%$ & $56,2 \%$ & $57,7 \%$ & $55,7 \%$ \\
\hline
\end{tabular}

Tabla 5

Origen demanda/año

\begin{tabular}{|c|c|c|c|c|c|c|c|c|c|}
\hline & & & \multicolumn{6}{|l|}{ Año } & \multirow[t]{2}{*}{ Total } \\
\hline & & & 1998 & 1999 & 2000 & 2001 & 2002 & 2003 & \\
\hline \multirow{10}{*}{$\begin{array}{l}\text { Origen } \\
\text { demanda }\end{array}$} & \multirow{2}{*}{$\begin{array}{l}\text { Puerta de } \\
\text { Urgencias }\end{array}$} & Recuento & 318 & 295 & 304 & 262 & 276 & 687 & 2142 \\
\hline & & $\%$ de año & $78,9 \%$ & $77,6 \%$ & $76,2 \%$ & $74,2 \%$ & $79,8 \%$ & $80,2 \%$ & $78,2 \%$ \\
\hline & \multirow{2}{*}{$\begin{array}{l}\text { Observación } \\
\text { de Urgencias }\end{array}$} & Recuento & 20 & 28 & 18 & 15 & 18 & 44 & 143 \\
\hline & & $\%$ de año & $5,0 \%$ & $7,4 \%$ & $4,6 \%$ & $4,2 \%$ & $5,2 \%$ & $5,1 \%$ & $5,2 \%$ \\
\hline & \multirow{2}{*}{$\begin{array}{l}\text { Interconsulta de } \\
\text { otro servicio } \\
\text { hospitalario }\end{array}$} & Recuento & 32 & 18 & 23 & 31 & 26 & 52 & 182 \\
\hline & & $\%$ de año & $7,9 \%$ & $4,7 \%$ & $5,8 \%$ & $8,8 \%$ & $7,5 \%$ & $6,1 \%$ & $6,6 \%$ \\
\hline & \multirow{2}{*}{$\begin{array}{l}\text { Unidad de } \\
\text { hospitalización } \\
\text { Psiquiátrica }\end{array}$} & Recuento & 27 & 35 & 50 & 43 & 22 & 68 & 245 \\
\hline & & $\%$ de año & $6,7 \%$ & $9,2 \%$ & $12,5 \%$ & $12,2 \%$ & $6,4 \%$ & $7,9 \%$ & $8,9 \%$ \\
\hline & \multirow{2}{*}{$\begin{array}{l}\text { Consulta } \\
\text { telefónica }\end{array}$} & Recuento & 6 & 4 & 4 & 2 & 4 & 6 & 26 \\
\hline & & $\%$ de año & $1,5 \%$ & $1,1 \%$ & $1,0 \%$ & $0,6 \%$ & $1,2 \%$ & $0,7 \%$ & 0,9 \\
\hline \multicolumn{2}{|l|}{ Total } & Recuento & 403 & 380 & 399 & 353 & 346 & 857 & 2738 \\
\hline
\end{tabular}




\section{ORIGINALES Y REVISIONES}

Tabla 6

Motivo de consulta

\begin{tabular}{|c|c|c|c|}
\hline Motivo de consulta & Casos & $\begin{array}{l}\text { Porcentaje } \\
\text { de las respuestas }\end{array}$ & $\begin{array}{l}\text { Porcentaje } \\
\text { de los casos }\end{array}$ \\
\hline $\begin{array}{l}\text { Orgánica, estados confusionales agudos, } \\
\text { demencias, trastornos neurológicos }\end{array}$ & 92 & 3,0 & 3,3 \\
\hline $\begin{array}{l}\text { Estados delirantes alucinatorios agudos. } \\
\text { Descompensaciones de trastornos delirantes } \\
\text { y/o alucinatorios }\end{array}$ & 261 & 8,6 & 9,4 \\
\hline $\begin{array}{l}\text { Trastornos del estado de ánimo hacia la } \\
\text { depresión-melancolía }\end{array}$ & 155 & 5,1 & 5,6 \\
\hline $\begin{array}{l}\text { Trastornos del estado de ánimo hacia la euforia, } \\
\text { hipomanía, crisis maniaca }\end{array}$ & 50 & 1,6 & 1,8 \\
\hline $\begin{array}{l}\text { Trastornos de conducta (agitación hetero- } \\
\text { autoagresividad) en la situación que motiva la consulta }\end{array}$ & 316 & 10,4 & 11,4 \\
\hline $\begin{array}{l}\text { Intento de suicidio, ingesta medicamentosa, } \\
\text { autolesiones:.. }\end{array}$ & 506 & 16,7 & 18,3 \\
\hline Estados de ansiedad, crisis ataques de angustia & 313 & 10,3 & 11,3 \\
\hline Queja o trastorno somático como motivo de consulta & 116 & 3,8 & 4,2 \\
\hline Trastornos alimentarios anorexia/bulimia & 18 & 0,6 & 0,7 \\
\hline $\begin{array}{l}\text { Abuso o dependencia a sustancias como motivo } \\
\text { de consulta }\end{array}$ & 195 & 6,4 & 7,0 \\
\hline $\begin{array}{l}\text { Urgencias médicas por efectos secundarios a } \\
\text { psicofármacos }\end{array}$ & 41 & 1,4 & 1,5 \\
\hline $\begin{array}{l}\text { Demanda de revisión de tratamiento en pacientes } \\
\text { en tratamiento psiquiátrico como motivo de consulta. }\end{array}$ & 77 & 2,5 & 2,8 \\
\hline Motivos sociales & 84 & 2,8 & 3,0 \\
\hline Problemas de infancia y adolescencia & 12 & 0,4 & 0,4 \\
\hline $\begin{array}{l}\text { Trastornos adaptativos como reacción a } \\
\text { situaciones traumáticas }\end{array}$ & 67 & 2,2 & 2,4 \\
\hline Otros, desconocido & 733 & 24,1 & 26,5 \\
\hline Total & 3036 & 100,0 & 100,0 \\
\hline
\end{tabular}


Tabla 7

Diagnóstico sindrómico/año

\begin{tabular}{|c|c|c|c|c|c|c|c|c|c|}
\hline & & & \multicolumn{6}{|l|}{ Año } & \multirow[t]{2}{*}{ Total } \\
\hline & & & 1998 & 1999 & 2000 & 2001 & 2002 & 2003 & \\
\hline \multirow{18}{*}{$\begin{array}{l}\text { Diagnóstico } \\
\text { sindrómico }\end{array}$} & \multirow{2}{*}{$\begin{array}{l}\text { Trastornos } \\
\text { orgánicos }\end{array}$} & Recuento & 7 & 12 & 14 & 10 & 16 & 51 & 110 \\
\hline & & $\%$ de año & $1,7 \%$ & $3,1 \%$ & $3,5 \%$ & $2,8 \%$ & $4,6 \%$ & $5,8 \%$ & $4,0 \%$ \\
\hline & \multirow{2}{*}{$\begin{array}{l}\text { Delirante/aluci- } \\
\text { natorio trastornos } \\
\text { psicóticos }\end{array}$} & Recuento & 98 & 60 & 69 & 69 & 85 & 193 & 574 \\
\hline & & $\%$ de año & $24,3 \%$ & $15,7 \%$ & $17,3 \%$ & $19,2 \%$ & $24,2 \%$ & $22,1 \%$ & $20,7 \%$ \\
\hline & \multirow{2}{*}{$\begin{array}{l}\text { Trastornos } \\
\text { afectivos } \\
\text { maníaco-depresivos }\end{array}$} & Recuento & 36 & 59 & 65 & 23 & 23 & 87 & 293 \\
\hline & & $\%$ de año & $8,9 \%$ & $15,4 \%$ & $16,3 \%$ & $6,4 \%$ & $6,6 \%$ & $10,0 \%$ & $10,6 \%$ \\
\hline & \multirow{2}{*}{$\begin{array}{l}\text { Trastornos } \\
\text { neuróticos } \\
\text { ansiedad-depresión }\end{array}$} & Recuento & 55 & 23 & 17 & 73 & 52 & 186 & 406 \\
\hline & & $\%$ de año & $13,6 \%$ & $6,0 \%$ & $4,3 \%$ & $20,3 \%$ & $14,8 \%$ & $21,3 \%$ & 14,7 \\
\hline & \multirow{2}{*}{$\begin{array}{l}\text { Trastornos perso- } \\
\text { nalidad, de control } \\
\text { de impulsos }\end{array}$} & Recuento & 40 & 21 & 34 & 29 & 86 & 177 & 387 \\
\hline & & $\%$ de año & $9,9 \%$ & $5,5 \%$ & $8,5 \%$ & $8,1 \%$ & $24,5 \%$ & $20,3 \%$ & 14,0 \\
\hline & \multirow{2}{*}{$\begin{array}{l}\text { Trastornos } \\
\text { alimentación }\end{array}$} & Recuento & 5 & 2 & 4 & 3 & 4 & 10 & 28 \\
\hline & & $\%$ de año & $1,2 \%$ & $0,5 \%$ & $1,0 \%$ & $0,8 \%$ & $1,1 \%$ & $1,1 \%$ & $1,0 \%$ \\
\hline & \multirow{2}{*}{$\begin{array}{l}\text { Dependencia- } \\
\text { abuso de } \\
\text { sustancias }\end{array}$} & Recuento & 37 & 37 & 36 & 37 & 25 & 65 & 237 \\
\hline & & $\%$ de año & $9,2 \%$ & $9,7 \%$ & $9,0 \%$ & $10,3 \%$ & $7,1 \%$ & $7,4 \%$ & $8,6 \%$ \\
\hline & \multirow{2}{*}{$\begin{array}{l}\text { Deficiencia } \\
\text { mental }\end{array}$} & Recuento & 18 & 20 & 10 & 19 & 13 & 23 & 103 \\
\hline & & $\%$ de año & $4,5 \%$ & $5,2 \%$ & $2,5 \%$ & $5,3 \%$ & $3,7 \%$ & $2,6 \%$ & $3,7 \%$ \\
\hline & \multirow[t]{2}{*}{ Otros } & Recuento & 107 & 149 & 150 & 97 & 47 & 81 & 631 \\
\hline & & $\%$ de año & $26,6 \%$ & $38,9 \%$ & $37,6 \%$ & $26,9 \%$ & $13,4 \%$ & $9,3 \%$ & $22,8 \%$ \\
\hline Total & & Recuento & 403 & 383 & 399 & 360 & 351 & 873 & 2769 \\
\hline
\end{tabular}




\section{ORIGINALES Y REVISIONES}

Tabla 8

Destino del paciente

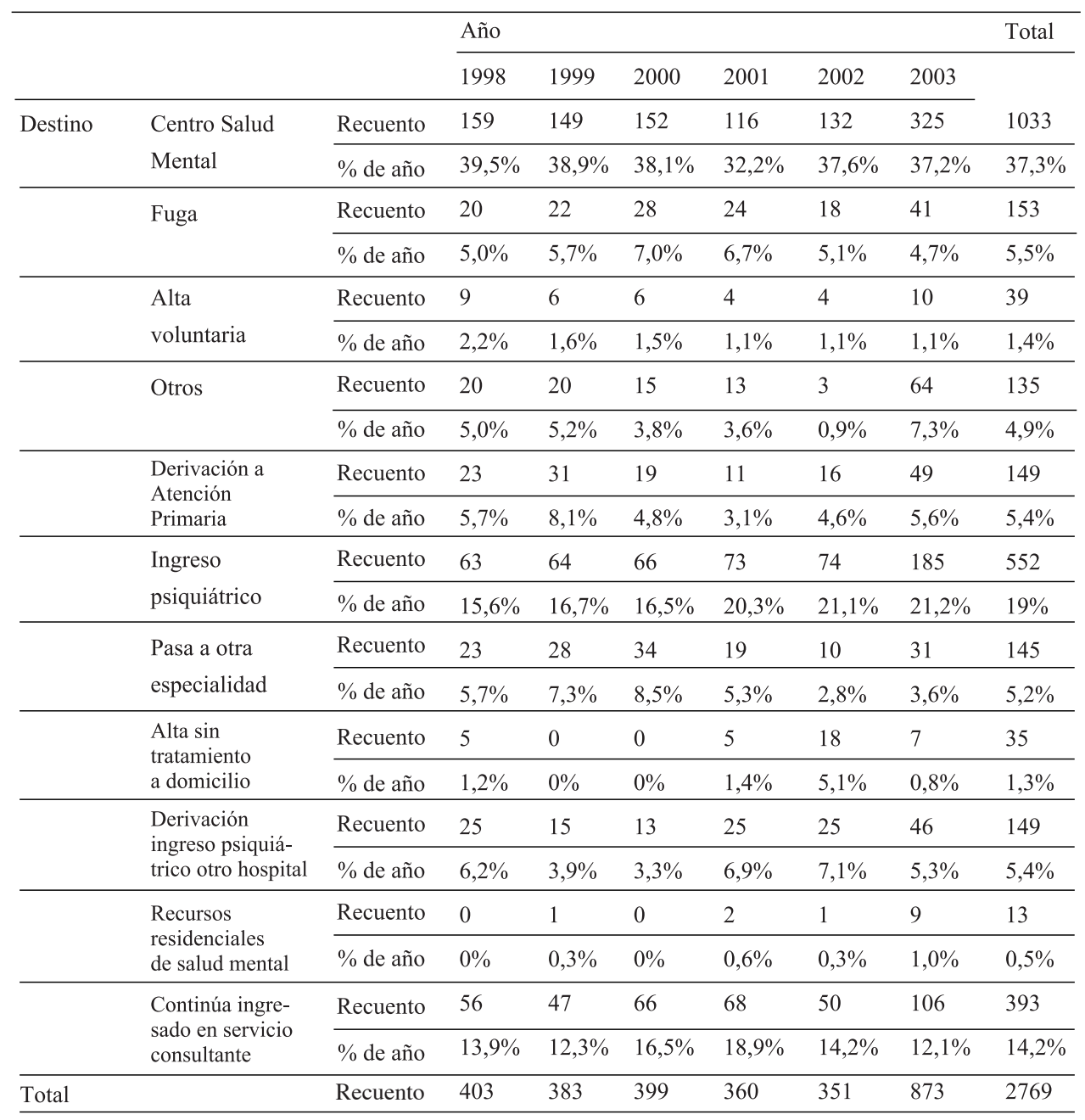

Tabla 9

Motivo de consulta/sexo

\begin{tabular}{lll}
\hline Motivo de consulta & $\%$ casos mujer & \% casos varones \\
\hline Trast. Conducta (agresividad) & 6,2 & 13,8 \\
\hline Intentos autolisis... & 24,4 & 12,2 \\
\hline Abuso drogas & 2,5 & 6,6 \\
\hline
\end{tabular}




\section{BIBLIOGRAFÍA:}

(1) Way, B.; Banks, S., Factors Related to Admission and Release Decisions in Psychiatric Emergency Services. Psychiatr Serv, 2001, February, 52, pp. 214-218.

(2) 2. Luque, A. Y Otros, Diferencias sociodemográficas y de utilización del servicio entre primeras consultas programadas y urgentes en un centro de salud mental, Psiquis, 2001, 22, 3, pp. 130-136.

(3) Mantrana Ridruejo, L., Y Otros, Factores asociados a la derivación urgente en un centro de salud mental, Actas Esp Psiquiatr, 2003, 31, 7, pp:16-22.

(4) Kung, S.; MrazeK, D.A., Psychiatric emergency department visits on full-moon nights, Psychiatr Serv., 2005, Feb, 56, 2,pp. 221-2.

(5) Olfson, M.; Y Otros, Emergency Treatment of Young People Following Deliberate Self-harm Arch Gen Psychiatry, 62, pp.1122-1128.

(6) Lozano Serrano C., Y Otros, Estudio descriptivo del parasuicidio en las urgencias psiquiátricas, Revista de la Asociación Española de Neuropsiquiatría, 2004, sep, 91, pp.11-22.

(7) Rubio Domínguez E; Y Otros, Intento y riesgo suicida en la emergencia psiquiátrica. Alcmeón, Revista Argentina de Clínica Neuropsiquiátrica, 2004, abr; 11,2.

(8) Rubio Domínguez E; Y Otros, Problemática de los auxilios en emergencias psiquiátricas, Alcmeón Revista Argentina de Clínica Neuropsiquiátrica, 2001, marzo, 9, 4.

(9) MCNiel D.E.; BINDER, R.L.., Psychiatric emergency service use and homelessness, mental disorder, and violence, Psychiatr Serv., 2005, Jun; 56, 6,pp.699-704.

(10) Bruffaerts R.; Sabbe, M.; Demyttenaere, K., Attenders of a university hospital psychiatric emergency service in Belgium - general characteristics and gender differences, Soc Psychiatry Psychiatry Epidemiol., 2004, Feb, 39, 2, pp.146-53.

(11) Oster, A.;, Bernbaum, S.; Patten, S., Determinants of violence in the psychiatric emergency service. Canadian Medical Association Journal, 2001 January, 9, 164, 1, pp.32-33.

(12) Lam, J.N.; MCNiel, D.E.; Binder, R.L., The relationship between patients' gender and violence leading to staff injuries, Psychiatr Serv., 2000, Sep, 51, 9,pp.1167-70

(13) Ricardo Teijeiro, C.; Westerkamp, D.; KoK, H., Cambios en la demanda asistencial de urgencias psiquiátricas, interpsiquis. 2003; http://www.psiquiatria.com/congreso, http://www.psiquiatria.com/interpsiquis2003/9934

(14) Torres Hernández, P.; Y Otros. Estudio de los trastornos de personalidad en urgencias psiquiátricas, Anales de Psiquiatría. 2004, ago; 20, 7, pp.294-298

(15) De Pablo Rabasso J; Y Otros. Appropriateness and complexity of the psychiatric emergencies in a General hospital, Medicina Clínica, 2003,. 121, 17, pp. 650-2.

(16) D. BARCIA; M E. Ruzz. Urgencia psiquiátrica y crisis psicosocial, Archivos de Psiquiatría, 2005, 68, 11, pp.1-30. 
ORIGINALES Y REVISIONES

* Conde Díaz Manuel. Psiquiatra. Esteban Ortega Cristina. Psiquiatra. Rosado Jimenez Lourdes. Psiquiatra. Barroso Peñalver María Dolores. Psiquiatra. Romero Gonzalez Sara. Psiquiatra

Área Salud Mental, Hospital Universitario Virgen del Rocío Sevilla. Servicio Andaluz de Salud.

\section{Correspondencia:}

Dr. Manuel Conde Díaz, Centro de Salud Mental Sur, HHUU Virgen del Rocío. C/ Domingo Tejera s/n, 41013-Sevilla. Tfno: 9550134 94. Correo electrónico: manuel.conde.diaz.sspa@juntadeandalucia.es

** Recibido: 17/07/2008. 